\title{
Comprehensive methods for measuring regional multidimensional development and their applications in China
}

\author{
XU Yong ${ }^{1,2}$, DUAN Jian ${ }^{1,2},{ }^{*} X U$ Xiaoren $^{3}$ \\ 1. Institute of Geographic Sciences and Natural Resources Research, CAS, Beijing 100101, China \\ 2. University of Chinese Academy of Sciences, Beijing 100049, China; \\ 3. Shandong Provincial Key Laboratory of Water and Soil Conservation and Environmental Protection, College \\ of Resources and Environment, Linyi University, Linyi 276000, Shandong, China
}

\begin{abstract}
National and international research on regional development has matured from the use of single elements and indicators to the application of comprehensive multi-element and multi-indicator measures. We selected 12 indicators from six dimensions for analysis in this study, including income, consumption, education, population urbanization, traffic, and indoor living facilities. We then proposed the polyhedron method to comprehensively measure levels of regional multidimensional development. We also enhanced the polygon and vector sum methods to render them more suitable for studying the status of regional multidimensional development. Finally, we measured levels of regional multidimensional development at county, city, and provincial scales across China and analyzed spatial differences using the three methods above and the weighted sum method applied widely. The results of this study reveal the presence of remarkable regional differences at the county scale across China in terms of single and multidimensional levels of regional development. Analyses show that values of the regional multidimensional development index (RMDI) are high in eastern coastal areas, intermediate in the midlands and in northern border regions, and low in the southwest and in western border regions. Districts characterized by enhanced and the highest levels of this index are distributed in eastern coastal areas, including cities in central and western regions, as well as areas characterized by the development of energy and mineral resources. The regional distribution of reduced and the lowest levels of this index is consistent with concentrations of areas that have always been impoverished. Correlation analyses of the results generated by the four methods at provincial, city, and county scales show that all are equivalent in practical application and can be used to generate satisfactory measures for regional multidimensional development. Additional correlation analyses between RMDI values calculated using the polyhedron method and per capita gross domestic product (GDP) demonstrate that the latter is not a meaningful proxy for the level of regional multidimensional development.
\end{abstract}

Keywords: regional multidimensional development; comprehensive methods; polyhedron method; polygon method; vector sum method; weighted sum method; China

Received: 2017-06-13 Accepted: 2017-12-20

Foundation: National Natural Science Foundation of China, No.41171449; Knowledge Innovation Project of the Chinese Academy of Sciences, No.KZZD-EW-06

Author: Xu Yong, Professor, specialized in regional sustainable development and human-environment interactions.

E-mail: xuy@igsnrr.ac.cn

"Corresponding author: Xu Xiaoren, PhD, E-mail: xuxiaoren@lyu.edu.cn 


\section{Introduction}

Regional development is a core research field in economic geography and is key to our understanding of local areas (Li et al., 2016; Liu et al., 2016). As a result, the quantitative assessment of regional development level and the differences between them have become hugely debated issues for governments and within academia (Salvati et al., 2016; United Nations, 1954). In the early studies, regional development levels or gaps were measured using one-dimensional economic indicators, including national income, gross national product, and GDP (Galbraith, 1958; Mishan, 1967). However, as people have become increasingly aware of the importance of health, education, and public welfare, subsequent research has not been constrained by economic income comparisons and has attached more importance to the integration of composite indexes and the use of social indicators (Sen, 1991).

Morris (1978) proposed the concept of a physical quality of life index (PQLI), encompassing rates of infant mortality and literacy of people over 15 years old as well as life expectancy. He advocated use of the equally weighted sum method to calculate this composite index (Morris, 1978); thus, PQLI is very simple, easy to understand, and can be used as a proxy for the level of public health care and the living standards of residents within a whole country or specific region (Liang, 1994). Although this index is not able to provide a marker for the whole of social welfare and couldn't be used as a reflection of the level of regional development, it nevertheless has enabled new research directions in the use of multidimensional comprehensive measures. In light of the limitations of using GDP to measure the level of regional development, a 1990 human development report issued by the United Nations Development Program (UNDP) proposed use of the Human Development Index (HDI), a measure that was created by the economists Amartya Sen (1991) and Mahbubul Haq (1999). This index encompasses health, education, and national income elements, and contains indicators of per capita GDP, life expectancy, adult literacy rate, and the gross enrolment ratio (Mahbub, 1999; UNDP, 1990). The HDI is calculated using the equally weighted sum method and can be used to measure the level of regional multidimensional development since it is not only based upon PQLI, but also uses per capita GDP as a supplementary indicator.

Both the HDI and PQLI are general methods that have been continually improved and perfected since they were first proposed (Grimm et al., 2008; Noorbakhsh, 1998; Nussbaum, 2000). Indeed, a selection of such indicators are also applied outside of economics and social science in areas related to the environment and management (Barro and Lee, 2010; Ediger and Tatlidil, 2006; Fukuda-Parr et al., 2010). As a result, studies have extended from treating countries as their basic units to consideration of regions and other subsidiary scales (Pradhan and Sanyal, 2011; Silva, 2014). In short, international research on regional development has evolved from the use of one-dimensional measurements towards the application of comprehensive multidimensional techniques.

Because of the confidentiality of economic and social data, research on Chinese regional development was rare prior to the 1980s, when large-scale studies were carried out for the first time. On the basis of existing literature and results, it is clear that a great deal of research carried out within China initially applied one-dimensional indexes, such as GDP and household income, to measure regional gaps in development and to analyze the influencing factors. Some of the methods initially applied include the Gini coefficient, the Theil index, 
the integrated entropy index, and the coefficient of variation (Lin and Liu, 2003; Liu, 2006; Wang and Fan, 2004).

A small number of research results based on the use of comprehensive measures have begun to appear in recent years in a number of publications and variable progress has been made. For example, by carrying out screening, classification, and an attribute analysis of elements of regional development, the 'regional development gap assessment and regulation' project team of Chinese Academy of Sciences constructed a macroscopic-microcosmic indicator system that is aimed at measuring the quality of people's livelihoods (Xu and Fan, 2014). Although just a handful of multidimensional measure-based methods for assessing regional development are available, the weighted sum, vector sum, and polygon methods have been applied successfully in related fields. Of these, the HDI is a typical weighted sum method that has been frequently applied in domestic research to the extent that its indicators and algorithm have been continuously revised and improved (Fu et al., 2014; Lu, 2012). In addition, research on major function-oriented zoning has led to application of the vector sum method to evaluate the suitability of land for development and protection, applying the difference values between the vector sums of the two indexes as composites (Fan, 2015). Liu and $\mathrm{Xu}$ (2016) reported the results of county-level determination of multidimensional poverty in rural China by establishing a multi-indicator system and a more precise method of integration based on a vulnerability and sustainable livelihood framework. This approach is one example of a typical application of the polygon area method for the integration of multi-indicators (Liu and $\mathrm{Xu}, 2016$ ). In summary, both gaps and levels of regional development have been measured to date within China by mainly applying one-dimensional indexes, although the use of multidimensional integration methods in other related fields can be taken as references.

Building on the use of multidimensional integration methods as references, and given data accessibility, we selected 12 indicators from six dimensions for use in this study, including income, consumption, education, population urbanization, traffic, and indoor living facilities. We then proposed the polyhedron method and enhanced the polygon and vector sum method, combined with the weighted sum method applied widely, to comprehensively measure the levels of regional multidimensional development and gaps at county, city, and provincial scales, based on the indicators of six dimensions. This study has four main aims, including initially to establish and modify the use of comprehensive measurements methods to study gaps in regional multidimensional development. Secondly, this study aims to analyze spatial differences in Chinese regional development from integrated multi-element and multi-indicator perspectives. Thirdly, we compare the results generated using different methods to provide a basis for the future selection of approaches in this research field. Finally, we consider whether per capita GDP can be used to replace the composite index by conducting a series of correlation analyses.

\section{Materials and methods}

\subsection{An indicator system for measuring levels of regional multidimensional development}

Two kinds of concepts in regional development are recognized that differ in terms of their constituent parts. In the first case, regional development can be used to refer just to eco- 
nomic development, while the second concept refers to multidimensional development that supplements elements of social development and human settlement. Thus, regional multidimensional development is not just concerned with the development of a region in terms of economy, society, and human settlements, but can also refer to the concept of overall progress. This overall level can be characterized by use of the regional multidimensional development index (RMDI). As a result, regional multidimensional development has five characteristic attributes, including regional properties, objectivity, development, and integrality, as well as variability. Regional properties refer to the fact that the objectives of a study are always constrained within certain borders that could be administrative or natural, while objectivity refers to the constituent elements that comprise economy, society, and human settlements. Development compares the stage that is characteristic of the constituent elements that comprise the dynamic development process, while integrality refers to studies of composite indexes or comparisons between different RMDI indicators in different regions. The meaning of variability is broader than the meaning of gaps and can be expressed quantitatively.

Building on the use of these constituent elements of regional multidimensional development, including economy, society, and human settlements, and taking into account literature that is related to the use of indicator systems (e.g., Fang and Mao, 1999; Xu and Fan, 2014), this study establishes an indicator system for use as a multidimensional measure (Table 1). The level of economic development was characterized using indicators of residential income and consumption, while indicators of the level of labor-force education and the rate of population urbanization were used to reflect the level of social development. We chose to use indicators of traffic development and the allocation of indoor living facilities as proxies for the quality of human settlements.

Table 1 Indicators of regional multidimensional development

\begin{tabular}{|c|c|c|c|}
\hline Element & $\begin{array}{l}\text { Feature indicator/ } \\
\text { Dimension }\end{array}$ & Attribute indicator & Equation \\
\hline \multirow[t]{4}{*}{ Economy } & $\begin{array}{l}\text { Level of residential } \\
\text { income (I) }\end{array}$ & $\begin{array}{l}\text { Per capita disposable income of urban } \\
\text { households }\left(\mathrm{I}_{\mathrm{C}}\right)\end{array}$ & $I=I_{C} \times \beta+I_{R} \times(1-\beta)+I_{1}$ \\
\hline & & Per capita net income of rural households $\left(I_{R}\right)$ & \\
\hline & & Per capita local financial budget revenue $\left(\mathrm{I}_{\mathrm{F}}\right)$ & \\
\hline & $\begin{array}{l}\text { Level of residential } \\
\text { consumption }(\mathrm{C})\end{array}$ & $\begin{array}{l}\text { Per capita total retail sales of urban and rural } \\
\text { consumer goods }\end{array}$ & \\
\hline \multirow[t]{2}{*}{ Society } & $\begin{array}{l}\text { Level of } \\
\text { labor-force } \\
\text { education }(E)\end{array}$ & $\begin{array}{l}\text { Average number of education years for pop- } \\
\text { ulation of school age }\end{array}$ & \\
\hline & $\begin{array}{l}\text { Level of population } \\
\text { urbanization }(U)\end{array}$ & Rate of population urbanization $(\beta)$ & \\
\hline \multirow{5}{*}{$\begin{array}{l}\text { Human } \\
\text { settlement }\end{array}$} & Allocation level of & Allocation rate for indoor tap water $\left(\mathrm{F}_{\mathrm{W}}\right)$ & $F=1 / 4 \times\left(F_{W}+F_{K}+F_{T}+F_{B}\right)$ \\
\hline & indoor living & Allocation rate for indoor kitchens $\left(\mathrm{F}_{\mathrm{K}}\right)$ & Values for indicators have \\
\hline & facilities $(F)$ & $\begin{array}{l}\text { Allocation rate for indoor toilets }\left(\mathrm{F}_{\mathrm{T}}\right) \\
\text { Allocation rate for indoor bathrooms }\left(\mathrm{F}_{\mathrm{B}}\right)\end{array}$ & been normalized \\
\hline & Level of traffic & Proportion of traffic land to total land $\left(\mathrm{T}_{\mathrm{P}}\right)$ & $T=1 / 2 \times\left(T_{P}+T_{D}\right)$ \\
\hline & development $(\mathrm{T})$ & Traffic Dominance $\left(\mathrm{T}_{\mathrm{D}}\right)$ (Jin et al., 2008) & $\begin{array}{l}\text { Values for indicators have } \\
\text { been normalized }\end{array}$ \\
\hline
\end{tabular}

\subsection{Methods for measuring levels of regional multidimensional development}

(1) The polyhedron method. This method uses the length of a line between a fixed point 
(the origin) and vertices as a value for each indicator and calculates the volume of a polyhedron, the RMDI. This equates to an octahedron (Figure 1) composed of six indicators (or dimensions), with $O$ as the original point, and $O E, O U, O T$, and $O F$ denoting the level of labor-force education, population urbanization, traffic development, and the allocation level of indoor living facilities, respectively. Thus, in terms of the four feature indicators (dimensions) used in this study, the angles between two adjacent dimensions would be $\alpha$ ( $\alpha=$ $360^{\circ} / 4$ ), while $O I$ and $O C$ are perpendicular to the quadrilateral EUTF, and denote the levels of residential income and consumption, respectively. The volume of the octahedron can therefore be defined as $R M D I_{-}$, as follows:

$$
R M D I_{-V}=\frac{1}{6} \sin \alpha\left(a_{E} \times a_{U}+a_{U} \times a_{T}+a_{T} \times a_{F}+a_{F} \times a_{E}\right) \times\left(a_{I}+a_{C}\right)
$$

In this expression, $a_{E}$ denotes the level of labor-force education, while $a_{U}$ refers to the level of population urbanization, $a_{T}$ denotes the level of traffic development, $a_{F}$ denotes the allocation level of indoor living facilities, $a_{I}$ refers to the level of residential income, and $a_{C}$ denotes the level of residential consumption. The values for all indicators were normalized in this study.

(2) The polygon method. Two kinds of polygon methods are available, utilizing either sequential or full permutation. In the case of the former, a polygon comprises concurrent lines based on a fixed point. Each line represents a specific indicator, with lengths denoting values. Thus, the area of the polygon area serves as a composite index that can be calculated by summing adjacent triangular areas in order. This approach has a number of advantages when compared to full permutation (Liu and $\mathrm{Xu}, 2016$ ), including that since the length of each line represents a specific indicator value, it is simple to use graphs to compare measured units.

This calculation method therefore comprises a hexagon (Figure 2) that is composed of six feature indicators (dimensions), including the original point, $O$, alongside $O I, O C, O E, O U$, $O T$, and $O F$, which denote the level of residential income, residential consumption, labor-force education, population urbanization, traffic development, and the allocation level of indoor living facilities, respectively. Thus, the angles between two nearby dimensions are $a$

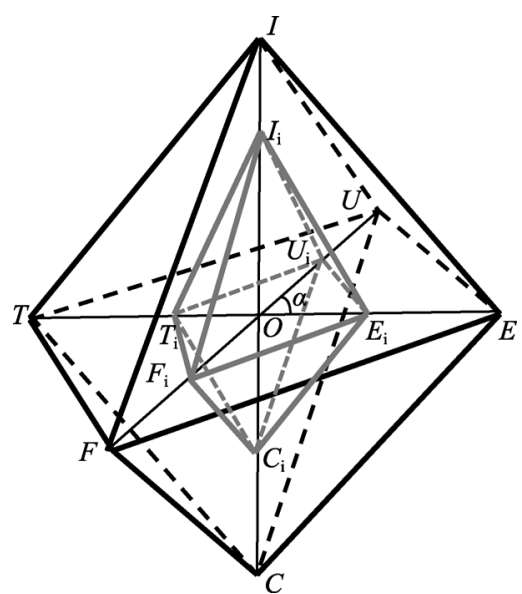

Figure 1 Sketches of the octahedron method for calculating the RMDI

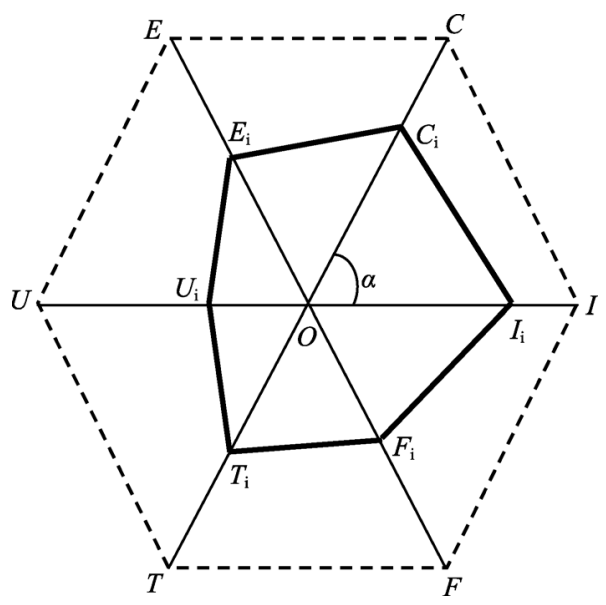

Figure 2 Sketches of the hexagon method for calculating the RMDI 
$\left(a=360^{\circ} / 6\right)$ and the area of the hexagon is defined as $R M D I_{-}$, as follows:

$$
R M D I_{-A}=1 / 2 \times \sin \alpha \times\left(a_{I} \times a_{C}+a_{C} \times a_{E}+a_{E} \times a_{U}+a_{U} \times a_{T}+a_{T} \times a_{F}+a_{F} \times a_{I}\right)
$$

The notations used in this case are the same as those used in Equation (1).

(3) The vector sum method. Because each indicator in this method is taken to be a vector, each module corresponds with an indicator value. Thus, the vector sum is defined as $R M D I \_$, simpler than either the polyhedron or polygon method, as follows:

$$
R M D I_{-M}=\sqrt{a_{I}^{2}+a_{C}^{2}+a_{E}^{2}+a_{U}^{2}+a_{T}^{2}+a_{F}^{2}}
$$

The notations used in this case are the same as those used in Equation (1).

(4) The weighted sum method. This method is commonly applied to calculate a RDM and includes both the HDI and the PQLI. In this case, the RMDI_W is calculated as follows:

$$
R M D I_{-W}=1 / 6 \times\left(a_{I}+a_{C}+a_{E}+a_{U}+a_{T}+a_{F}\right)
$$

The notations used in this case are the same as those used in Equation (1).

\subsection{Data and resources}

This study incorporates data from 12 indicators that cover the whole of China at the provincial, city, and county levels, with the exception of Hong Kong, Macao, and Taiwan. Data for the per capita disposable income of urban households, the per capita net income of rural households, local financial budget revenue, and the total retail sales of urban and rural consumer goods were extracted from the China Statistical Yearbook for Regional Economy 2011 (note that data for Tibet and Xinjiang are absent from this compilation). Data for average number of education years for population of school age, rate of population urbanization, and indoor living facilities (e.g., the presence of showers, kitchens, and tap water) were extracted from the 2010 population census. Thus, the allocation rate for each type of facility is the proportion of households that own a particular facility compared to the overall number of households sampled in a specific region. Sampled households account for $10 \%$ of the total families in a region, while the county boundaries applied are based on a 2004 map provided by the National Geomatics Center of China. Data for transportation areas were taken from the Ministry of Land and Resources for 2008, while traffic dominance data come from the Major Function Oriented Regionalization of China (The State Council, 2010). Income and consumption data for Tibet were provided by the local statistical bureau, while data for the cities of Shihezi, Alaer, Tumushuke, and Wujiaqu in Xinjiang come from their respective 12th Five-Year Plan for National Economic and Social Development.

\section{Results}

\subsection{Single dimension analysis}

We used the maximum standardization method to normalize feature indicator values for levels of residential income, residential consumption, the number of education years for population of school age (15-69), and rate of population urbanization to between 0 and 1 . Thus, applying the natural fracture method in the software ARCGIS, normalized indicator values for our four dimensions were then combined with those for the normalized level of traffic development and allocation of indoor living facilities and divided into five county level categories across China. These categories are referred to as highest, higher, medium, lower, and lowest (Figure 3 and Table 2). 

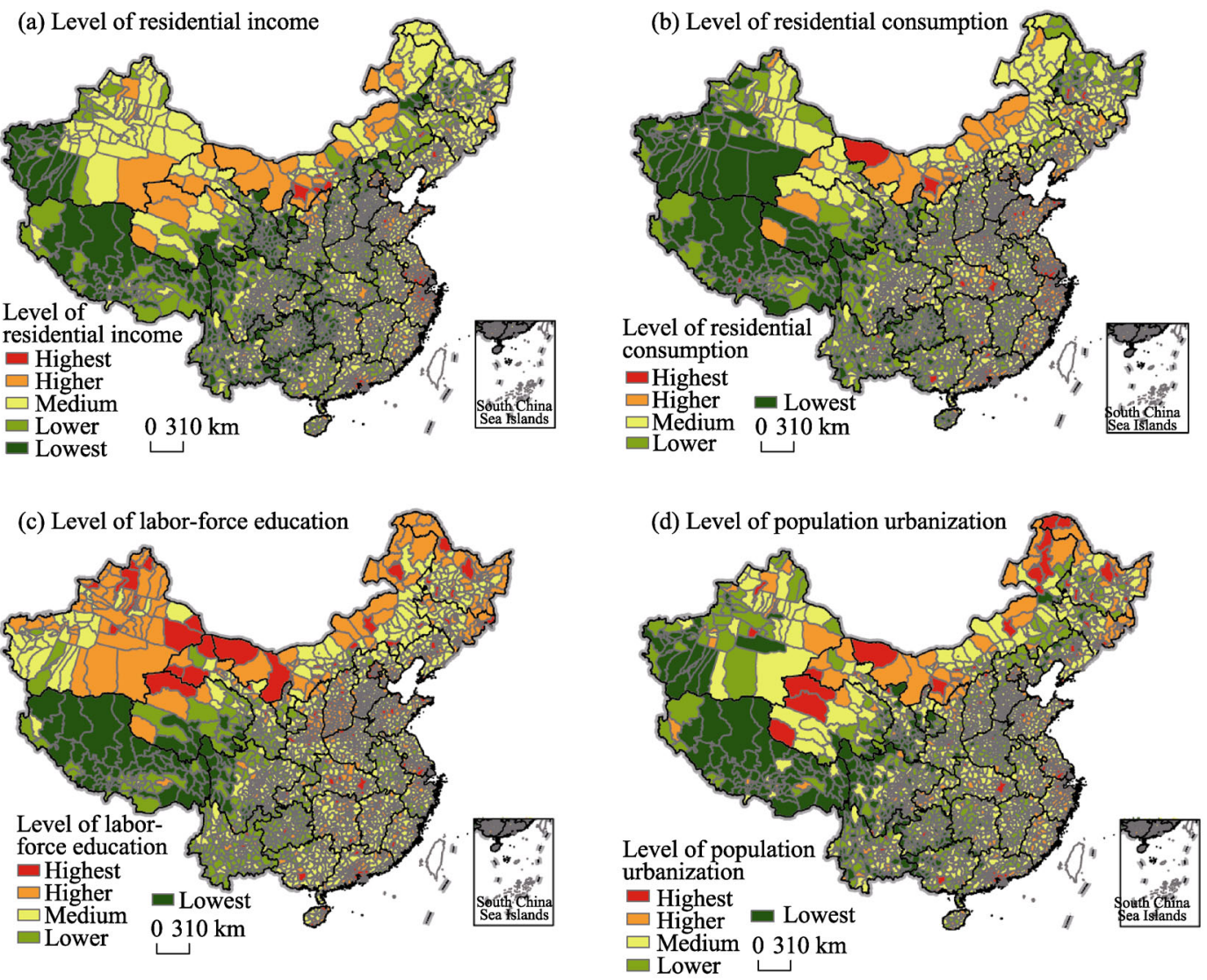

(e) Allocation level of indoor living facilities

(f) Level of traffic development
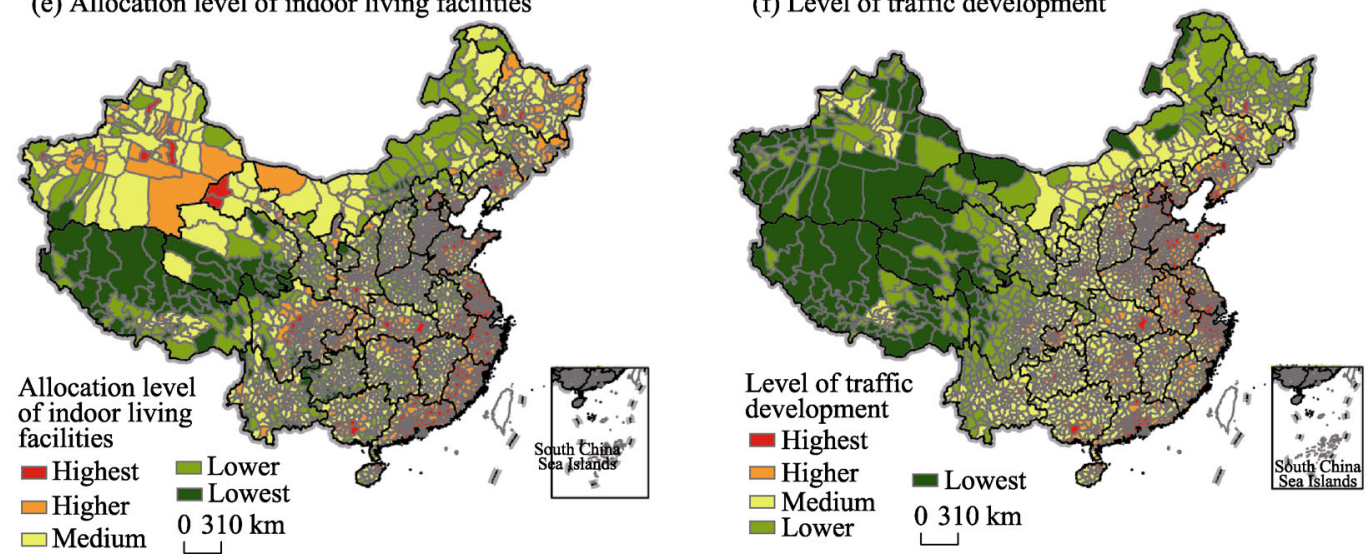

Figure 3 Single-dimension classification for China's regional development in 2010

The results of this analysis show that values of residential income are high in eastern coastal and northern border regions, but are low in the mountainous regions of western China (Figure 3a). Data also show that the Yangtze and Pearl river deltas, the Beijing-Tianjin region, and the cities of Hohhot and Ordos in Inner Mongolia are characterized by the highest levels of residential income and are also surrounded by areas where this measure is higher. In addition, coastal and central parts of Shandong, western Inner Mongolia and the Greater Khingan Mountains, as well as adjoining areas of Xinjiang, Qinghai, and Gansu, and 
Table 2 Single-dimension classification for China's regional development in 2010

\begin{tabular}{|c|c|c|c|c|}
\hline Indicator/dimension & Rank & Range & Number of counties & Average value \\
\hline \multirow{5}{*}{$\begin{array}{l}\text { Level of residential } \\
\text { income }\end{array}$} & Highest & $0.39-1$ & 103 & 0.49 \\
\hline & Higher & $0.25-0.39$ & 305 & 0.3 \\
\hline & Medium & $0.14-0.25$ & 993 & 0.18 \\
\hline & Lower & $0.1-0.14$ & 578 & 0.12 \\
\hline & Lowest & $0-0.1$ & 397 & 0.08 \\
\hline \multirow{5}{*}{$\begin{array}{l}\text { Level of residential } \\
\text { consumption }\end{array}$} & Highest & $0.15-1$ & 153 & 0.229 \\
\hline & Higher & $0.08-0.15$ & 405 & 0.106 \\
\hline & Medium & $0.035-0.08$ & 1,006 & 0.053 \\
\hline & Lower & $0.018-0.035$ & 563 & 0.027 \\
\hline & Lowest & $0-0.018$ & 249 & 0.013 \\
\hline \multirow{5}{*}{$\begin{array}{l}\text { Level of labor-force } \\
\text { education }\end{array}$} & Highest & $0.75-1$ & 259 & 0.81 \\
\hline & Higher & $0.68-0.75$ & 515 & 0.71 \\
\hline & Medium & $0.6-0.68$ & 1,114 & 0.64 \\
\hline & Lower & $0.4-0.6$ & 415 & 0.54 \\
\hline & Lowest & $0-0.4$ & 73 & 0.32 \\
\hline \multirow{5}{*}{$\begin{array}{l}\text { Level of population } \\
\text { urbanization }\end{array}$} & Highest & $0.75-1$ & 212 & 0.88 \\
\hline & Higher & $0.5-0.75$ & 318 & 0.60 \\
\hline & Medium & $0.3-0.5$ & 1,018 & 0.38 \\
\hline & Lower & $0.2-0.3$ & 562 & 0.26 \\
\hline & Lowest & $0-0.2$ & 266 & 0.14 \\
\hline \multirow{5}{*}{$\begin{array}{l}\text { Allocation level of } \\
\text { indoor living facilities }\end{array}$} & Highest & $0.8-1$ & 406 & 0.87 \\
\hline & Higher & $0.65-0.8$ & 648 & 0.72 \\
\hline & Medium & $0.45-0.65$ & 849 & 0.56 \\
\hline & Lower & $0.25-0.45$ & 370 & 0.37 \\
\hline & Lowest & $0-0.25$ & 103 & 0.15 \\
\hline \multirow{5}{*}{$\begin{array}{l}\text { Level of traffic } \\
\text { development }\end{array}$} & Highest & $0.42-1$ & 344 & 0.508 \\
\hline & Higher & $0.323-0.42$ & 610 & 0.346 \\
\hline & Medium & $0.2-0.323$ & 1,034 & 0.241 \\
\hline & Lower & $0.102-0.2$ & 280 & 0.110 \\
\hline & Lowest & $0-0.102$ & 108 & 0.101 \\
\hline
\end{tabular}

many other provincial capitals are also characterized by higher residential income levels. In contrast, distribution of the medium-value residential income group ranges widely from eastern China to the west, while the lower income group is mainly distributed across central and western China. Examples of the lower residential income group are predominantly concentrated in regions such as the eastern Greater Khingan and Taihang mountains, as well as in Henan and Anhui. In addition, southern Shaanxi, northern Sichuan, and southeastern Tibet are also characterized by lower residential income values, while the lowest value group is 
mainly located in western regions, including southern Xinjiang and Gansu, as well as on northern Tibetan Plateau and in the Wuling mountain areas in Guizhou and Hunan.

Results show that values of residential consumption are the highest on the eastern coastal China and the lowest in Northwest and Southwest China (Figure 3b). Data show that the highest values are seen in Beijing and Tianjin, in the Yangtze and Pearl river deltas, on the Shandong Peninsula, and in municipal districts, while the areas surrounding these centers of high residential consumption are also characterized by higher levels, patchily distributed across Northeast China, and in Hubei and Fujian. At the same time, medium values of residential consumption are widely distributed within each provincial unit, while lower values are seen on the Loess and Yunnan-Guizhou plateaus, in most parts of Guangxi, in western Hunan, southern Jiangxi, western Sichuan, in the southern border region of Tibet, and to the north of the Tianshan Mountains. The lowest levels of residential consumptions are seen across most parts of Tibet, and in southern Qinghai and Xinjiang.

Values for labor-force education are the highest in the north and east of China and are the lowest in the southwest (Figure 3c). The highest values of this index have a relatively fragmented distribution across wide swathes of China, characterizing urban districts as well as interconnected areas of Qinghai, Xinjiang, and Inner Mongolia. Higher values for labor-force education are also mainly characteristic of northern China; there is a continuous distribution of higher values in the northern border region, in most parts of Xinjiang, and in the central and western parts of the Loess Plateau. At the same time, higher values for labor-force education are patchily distributed across the middle reaches of the Yangtze River Basin, while medium values are widely distributed in eastern, central, and northeastern China. Lower values are mainly seen in western regions, including on the Yunnan-Guizhou Plateau, in adjoining areas of Gansu, Qinghai, and Sichuan, and in the southern border region of Tibet. Results show that the lowest values for this index are concentrated on the Qinghai-Tibet Plateau and in western Sichuan.

The spatial distribution of population urbanization values reveals the presence of a high-medium-low trend that spreads from the border regions of northern China and eastern coastal areas into central China, and then into Southwest China (Figure 3d). The highest values of population urbanization are scattered within the municipal districts of large cities and around the Greater Khingan Mountains, while higher values are mainly concentrated in the coastal areas and border regions of China. Data show that Beijing and Tianjin, the Yangtze and Pearl river deltas, the northeast, and border regions of Inner Mongolia are all characterized by higher values of population urbanization, while medium values are continuously distributed across the Huang-Huai-Hai Plain and middle-lower Yangtze Plains, within the Jiangnan hills, along the Hexi Corridor, and in eastern Xinjiang and parts of Qinghai. Lower values for population urbanization are widely distributed across China, while the lowest values for this index are mainly seen in the western regions, especially in Tibet, and in southwestern Xinjiang, northwestern Sichuan, and southern Gansu.

Values that denote the presence of indoor living facilities are the highest in the eastern coastal areas of China and along the Yangtze River, while this index is low in Southwest China and in western Inner Mongolia (Figure 3e). The highest values for indoor living facilities are seen in eastern coastal areas, and are also scattered along the Yangtze River, in Xinjiang and in the northeast of the country. Data show that the spatial distribution of higher values basically 
overlaps with the highest values, while medium values are more dispersed, concentrated in the northeast, along the Hexi Corridor, in Xinjiang, in western Inner Mongolia, and in western Qinghai, Jiangxi, Guangxi, and Yunnan. Lower values are found in central and western China; these levels of indoor living facilities have a sheet-like distribution across eastern Inner Mongolia, on the Loess Plateau, and in surrounding areas on the Qinghai-Tibet Plateau, as well as in Yunnan, Guangxi, Guizhou, and Henan. Data show that Southwest China is characterized by the lowest levels; these values are largely distributed across northern Tibet and the southern Qinghai Plateau, as well as patchily on the Yunnan-Guizhou and Loess plateaus.

Results for the spatial distribution of traffic development reveal the presence of a high-medium-low trend that spreads from the east to the west, with the lowest values located in the western regions along a line marked by Helanshan-Longmen-Jinghong (Figure 3f). The highest values of traffic development are seen within the urban agglomerations of Beijing-Tianjin-Hebei and in the Yangtze and Pearl river deltas, and also have a scattered distribution in eastern urban areas marked by the Helanshan-Longmen-Jinghong line. Values for the Huang-Huai-Hai and middle-lower Yangtze plains are also higher, and similar values are also seen along main railways, including the Beijing-Guangzhou, Beijing-Harbin, and Lanzhou-Lianyungang lines. Medium values for this index conform to a sheet-like distribution in areas that encompass northeastern-to-northern Inner Mongolia and the Loess Plateau, as well as the Sichuan Basin, Hubei, western Hunan, Guizhou, and Guangxi. Lower values are seen in Heilongjiang, in eastern Inner Mongolia, western Sichuan, western Yunnan, and in border regions adjacent to Gansu and Qinghai.

\subsection{Comprehensive multidimensional measures}

(1) A comprehensive measure based on the polyhedron method. Results based on use of the polyhedron method led to an average RMDI calculation of 0.0516 for the 2376 counties of China. Data also reveal remarkable regional differences at the county level countrywide; the highest composite index was 1.0357 for the Xicheng District of Beijing, while the lowest value was just 0.0003 for Zhongba County in Tibet. We then used the natural fracture method (Figure 4) to divide Chinese counties into five levels (i.e., highest, higher, medium, lower, and lowest) based on their RMDI values. Comparative results show that the coastal areas of eastern China mainly exhibit the highest and higher levels for this index, while medium values are found in the central region and in the border regions of northern China. In contrast, the southwest and western border regions of the country are mainly characterized by lower and

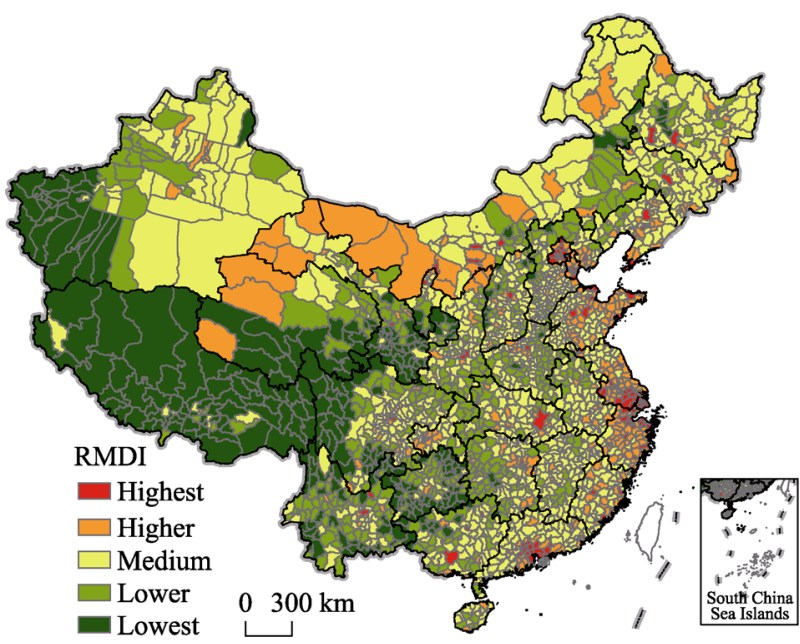

Figure 4 Spatial differences in the RMDI across China in 2010 the lowest levels. 
The highest composite index groupings are found in Beijing and Tianjin, in the Yangtze and Pearl river deltas, and in the eastern municipal districts delineated by the Helanshan-Longmen-Jinghong line. At the same time, regions characterized by higher composite index values are mainly distributed adjacent to areas that exhibit the highest values, including around Beijing-Tianjin-Hebei, in the Yangtze and Pearl river deltas, in Shandong and Qinghai, and in western Inner Mongolia. Examples of the medium composite index group are widely distributed across China, forming densely distributed clusters in Northeast, North China, and the middle-lower Yangtze plains, as well as in southeastern coastal areas, in the Sichuan Basin, and in the central and eastern parts of Xinjiang. Lower composite indexes tend to be relatively more concentrated in the central and western regions, including the eastern areas of the Greater Khingan Mountains, on the western Huang-Huai Plain, in mountainous regions of Wuling and Qinling-Daba, as well as in northern Hebei, Yunnan, Guizhou, Qinghai, and Xinjiang. Results show that the composite indexes for western regions are the lowest of all, encompassing the Qinghai-Tibet and Yunnan- Guizhou plateaus, as well as southwestern Xinjiang and southern Gansu.

The eastern coastal areas of China tend to exhibit highest, higher, and medium levels of development because of natural and geographic advantages as well as superior social and economic maturity. At the same time, western Inner Mongolia and Qinghai are also characterized by higher levels of development because of their sparse populations and economic expansion promoted by abundant energy resources. Key counties listed in the National Poverty Alleviation Plan and regions that have been impoverished over long periods of time tend to exhibit the lower and lowest values of composite indexes (Liu and $\mathrm{Xu}, 2016$ ).

(2) Comparative analysis of the four methods. As discussed above, we used SPSS software to carry out a series of correlation analyses between the results generated via the polyhedron, polygon, vector sum, and weighted sum methods. We performed comparative analyses at the county level and found the results of any two methods were correlated at a 0.01 significance level. We fitted data curves from the results of our four methods, using Excel, and generated power function relationships in each case (Figure 5). Although the results of these comparisons reveal a few differences between the results of the four methods, correlations are significant in all cases, with multiple correlation coefficients $\left(R^{2}\right)$ all higher than 0.9187 . These results demonstrate that the four methods applied in this study are all equivalent in terms of their practical application at the county level.

In order to further verify the feasibility of our four methods at different scales, we performed additional correlation analyses in SPSS and Excel using the same indicators and methods as before but at the levels of city and province (Figure 6 and Table 3). Curves fitted to results generated using the polyhedron and polygon methods at city and provincial levels both show similar linear function relationships and have multiple correlation coefficients $\left(R^{2}\right)$ of 0.93 and 0.9554 , respectively. Correlations between results generated using the vector sum and weighted sum methods reveal power function relationships at the city level and exponential function relationships at the provincial level, with $R^{2}$ of 0.9838 and 0.9747 , respectively. All other correlations conform to logarithmic function relationships, with $R^{2}$ all greater than 0.9204 . In other words, our results show that all four methods are equivalent in practical application at the city and provincial levels. 

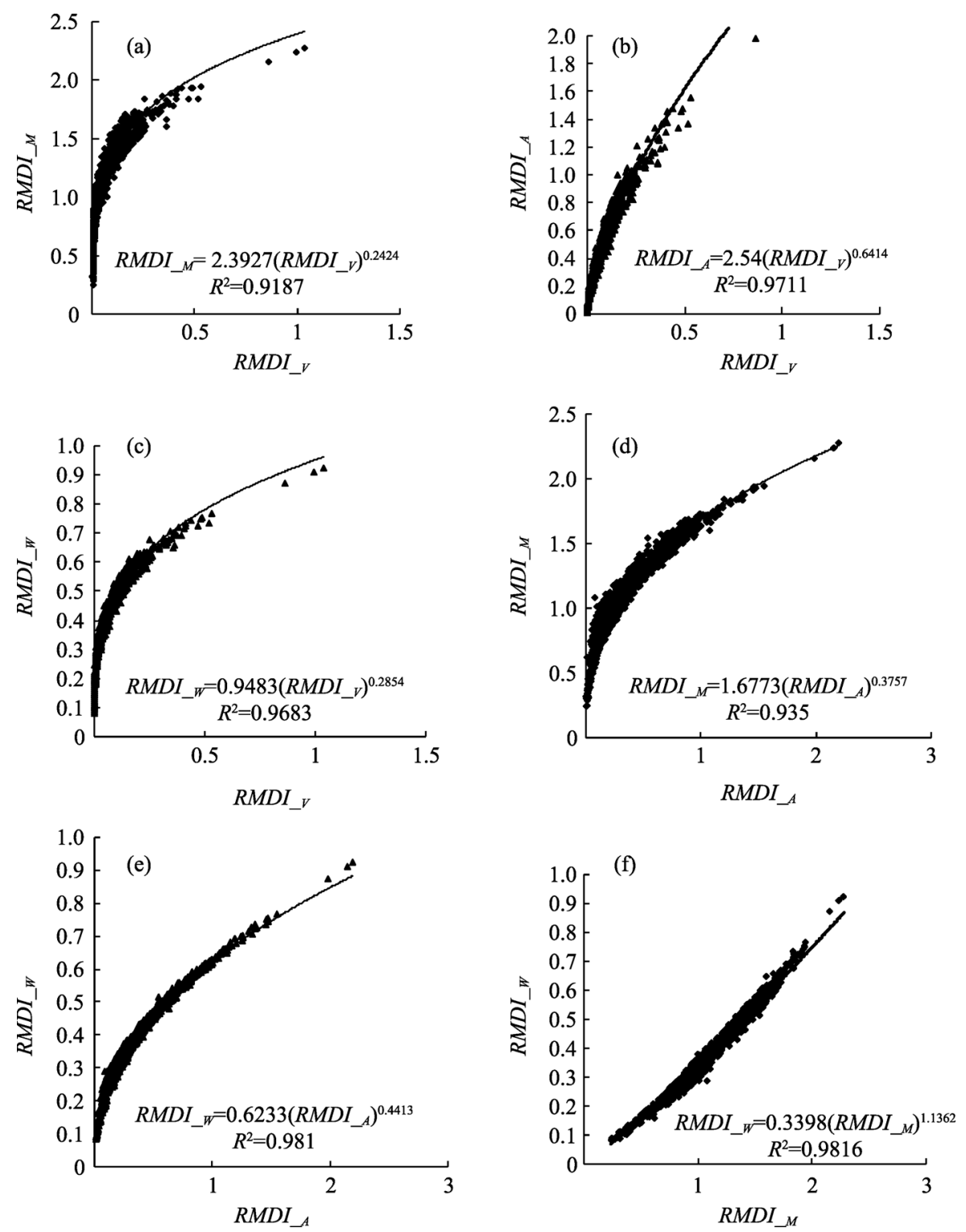

Figure 5 Curves fitted to results from the four measurement methods used in this study at the county level across China

\section{Discussion and conclusions}

\subsection{Discussion}

(1) Correlations between RMDI and per capita GDP. Although previous research has used per capita GDP as a proxy for regional development level, correlation analysis between RMDI calculated using the polyhedron method and per capita GDP at the county level suggest that the latter does not accurately reflect the level of regional multidimensional development. Our correlation analysis shows that there is no obvious relationship between these two variables; the maximum multiple correlation coefficient $\left(R^{2}\right)$ between the two was 

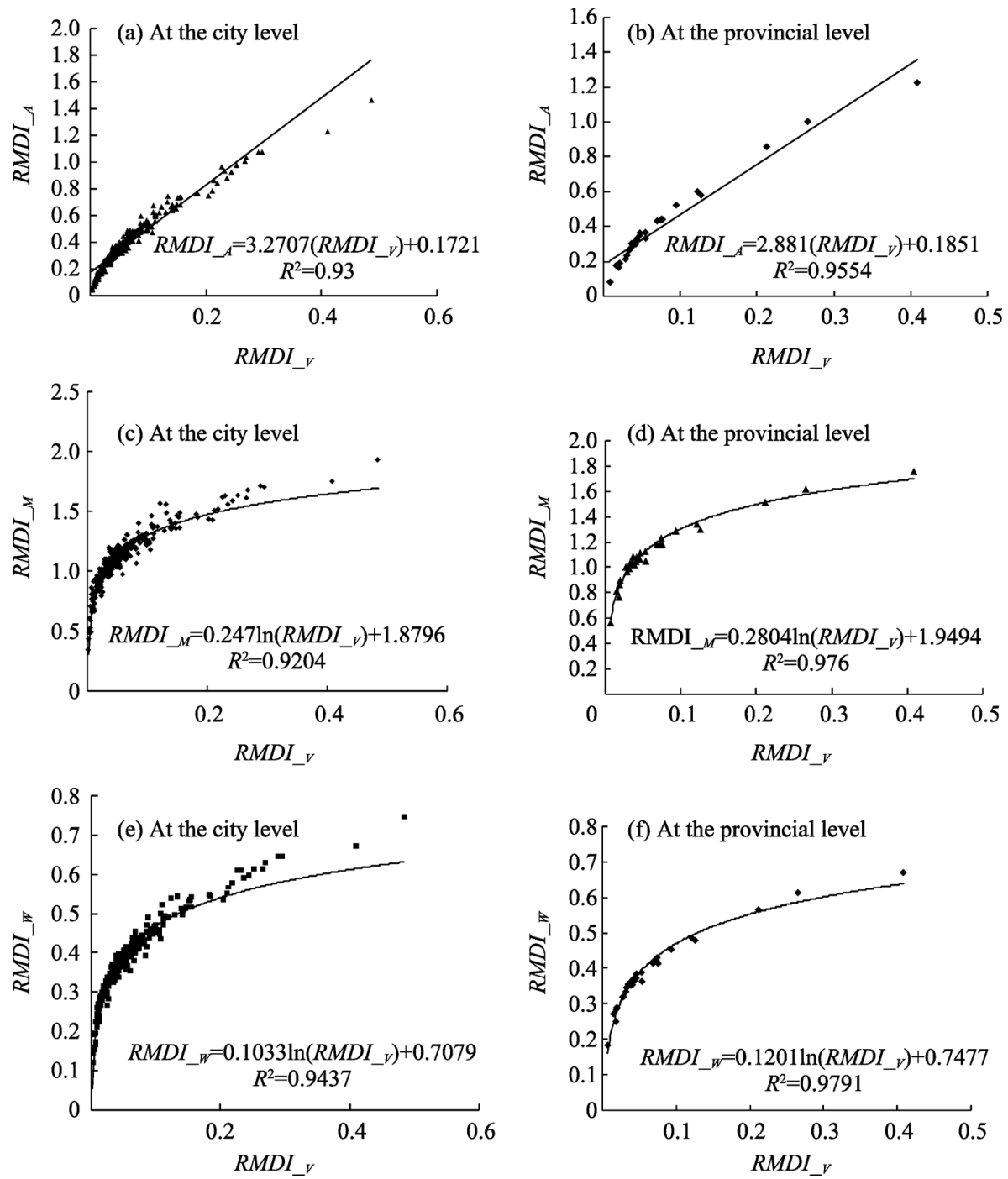

Figure 6 Curves fitted to results from the four measurement methods used in this study at the city and provincial levels across China

Table 3 Fitted equations and multiple correlation coefficients for the results of the four measurement methods used in this study at the provincial and city levels across China

\begin{tabular}{|c|c|c|c|}
\hline Level & Method & Equation & $R^{2}$ \\
\hline \multirow[t]{3}{*}{ City } & $R M D I_{-A}$ VS $R M D I_{-M}$ & $R M D I I_{M}=0.3902 \ln \left(R M D I_{-}\right)+1.5412$ & 0.9322 \\
\hline & $R M D I \_$VS $R M D I \_W$ & $R M D I \_W=0.1624 \ln \left(R M D I{ }_{A}\right)+0.5655$ & 0.9462 \\
\hline & $R M D I \_$VS RMDI_W & $R M D I_{-W}=0.3403\left(R M D I_{-M}\right)^{1.1574}$ & 0.9838 \\
\hline \multirow[t]{3}{*}{ Provincial } & $R M D I_{-A}$ VS $R M D I I_{M}$ & $R M D I_{-M}=0.427 \ln \left(R M D I_{-A}\right)+1.567$ & 0.9721 \\
\hline & $R M D I_{A}$ VS $R M D I I_{W}$ & $R M D I_{-W}=0.1821 \ln \left(R M D I_{A}\right)+0.583$ & 0.9664 \\
\hline & $R M D I \_$VS $R M D I \_W$ & $R M D I \_W=0.1143 \mathrm{e}^{1.0723\left(\text { RMDI } \_M\right)}$ & 0.9747 \\
\hline
\end{tabular}

0.4467 when plotted using a linear function (Figure 7). Correlation coefficients were even less significant when other functions were used. 
(2) It will be necessary to include ecological and environmental indicators in further studies. Much previous research has focussed on the question of how to incorporate ecological and environmental indicators within a comprehensive measurement index system and quantify them accurately. Quantifying these indicators has so far proved problematic, however, because ecological factors are diverse and there is often little to compare. In addition, air pollution is usually cross-regional, whereas water pollution is localized, soil pollution can be sophisticated, and the available environmental data used for

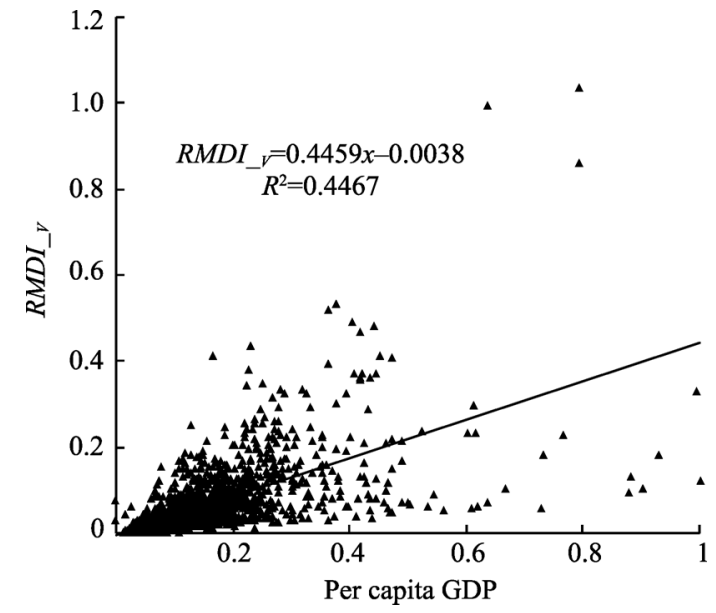

Figure 7 Lack of a correlation between the RMDI and per capita GDP monitoring remains incomplete. Therefore, although environmental and ecological indicators were not included in this study, it will be worthwhile to consider them in future research.

\subsection{Conclusions}

(1) Regional development research both nationally and internationally has developed from the use of trends in single elements and indicators towards the application of multi-element and multi-indicator comprehensive measures. Building on these developments, we selected 12 indicators from six dimensions, encompassing income, consumption, education, population urbanization, traffic, and indoor living facilities, and proposed the use of the polyhedron method to comprehensively measure levels of regional multidimensional development. We also enhanced the polygon and vector sum methods to render them more suitable for studying the status of regional multidimensional development. We present comprehensive measurements that quantify the level of regional multidimensional development at county, city, and provincial scales across China. We also analyze the spatial differences in these levels using these three methods, as well as the widely applied weighted sum approach. Our results show that while both the polyhedron and polygon methods are good for visual expression, the vector sum and weighted sum methods are relatively simpler.

(2) The results of this study reveal remarkable regional differences across China in the RMDI when calculated at the county level using the polyhedron method. Values for this index are high in eastern coastal areas, medium in the central regions and northern border regions, and low in the southwest and western border regions. Districts that are characterized by higher and the highest levels of the RMDI are distributed in eastern coastal areas, as well as in cities in central and western regions, and in areas where energy and mineral resources are being developed. Trends in the distribution of regions characterized by lower and the lowest levels of the RMDI coincide with regions of long-term poverty.

(3) We performed correlation analyses on the results of our four methods at provincial, city, and county levels. Results show that all four methods are equivalent to one another in practical application and can all be used to measure the RMDI. However, the lack of a cor- 
relation between RMDI calculated using the polyhedron method and per capita GDP at the county level indicates that the latter should not be used as a proxy for the level of regional multidimensional development.

\section{References}

Barro R J, Lee J W, 2010. A new data set of educational attainment in the World, 1950-2010. Journal of Development Economics, 104: 184-198.

Ediger V S, Tatlidil H, 2006. Energy as an indicator of human development: A statistical approach. The Journal of Energy and Development, 31(2): 213-232.

Fan Jie, 2015. Draft of major function oriented zoning of China. Acta Geographica Sinica, 70(2): 186-201. (in Chinese)

Fang Chuanglin, Mao Hanying, 1999. A system of indicators for regional development planning. Acta Geographica Sinica, 54(5): 410-419. (in Chinese)

$\mathrm{Fu}$ Wei, Zhao Junquan, Du Guozhen, 2014. The evaluation of resources sustainable utilization: Based on empirical analysis of resources welfare index. Journal of Natural Resources, 29(11): 1902-1914. (in Chinese)

Fukuda-Parr S, Lawson-Remer T, Randolph S, 2010. An index of economic and social rights fulfilment: Concept and methodology. Journal of Human Rights, 8(3): 195-221.

Galbraith J K, 1958. The Affluent Society. Boston: Houghton Mifflin Company.

Grimm M, Harttgen K, Klasen S et al., 2008. A human development index by income groups. World Development, 36(12): 2527-2546.

Jin F J, Wang C J, Li X W et al., 2010. China's regional transport dominance: Density, proximity, and accessibility. Journal of Geographical Sciences, 20(2): 295-309.

Li X J, Luo Q, Hai B B et al., 2016. Progress in the micro-perspective studies of economic geography in China since 1980s. Journal of Geographical Sciences, 26(8): 1041-1056.

Liang Hong, 1994. Applicable appraise for population quality life index. Chinese Journal of Social Medicine, 6: 12-13, 16. (in Chinese)

Lin Yifu, Liu Peilin, 2003. Chinese development strategy and economic convergence. Economic Research Journal, 3: 19-25, 89. (in Chinese)

Liu Hui, 2006. Regional inequality measurement: methods and evaluations. Geographical Research, 25(4): 710-718. (in Chinese)

Liu W D, Song Z Y, Liu Z G, 2016. Progress of economic greography in China's mainland since 2000. Journal of Geographical Sciences, 26(8): 1019-1040.

Liu Y H, Xu Y, 2015. A geographic identification of multidimensional poverty in rural China under the framework of sustainable livelihoods analysis. Applied Geography, 73: 62-76.

Lu Kangqiang, 2012. Component equilibrium: An improved composite method and its empirical test for HDI. Statistical Research, 29(10): 45-51. (in Chinese)

Mahbubul H, 1999. Reflections on Human Development. New York: Oxford University Press.

Mishan E J, 1967. The Cost of Economic Growth. London: Staples Press.

Morris M D, 1978. A physical quality of life index. Urban Ecology, 3(3): 225-240.

Noorbakhsh F, 1998. A modified Human development index. World Development, 26(3): 517-528.

Nussbaum M C, 2000. Women and Human Development: The Capabilities Approach. New York: Cambridge University Press.

Pradhan R, Sanyal G S, 2011. Good governance and human development: Evidence from Indian States. Journal of Social and Development Science, 1(1): 1-8.

Salvati L, Venanzoni G, Carlucci M, 2016. Towards (spatially) unbalanced development? A joint assessment of regional disparities in socioeconomic and territorial variables in Italy. Land Use Policy, 51: 229-235.

Sen A, 1991. Welfare, preference, and freedom. Journal of Econometrics, 50(1/2): 15-29.

Silva R, Ferreira-Lopes A, 2014. A regional development index for Portugal. Social Indicators Research, 118: 1055-1085.

UNDP, 1990. Human Development Report 1990. New York: Oxford University Press.

The State Council, 2010. National Major Function Zoning. The State Council Article No.46 in 2010. (in Chinese)

United Nations, 1954. Report on International Definition and Measurement of Standards and Levels of Living. New York: United Nations.

Wang Xiaolu, Fan Gang, 2004. Analysis on the regional disparity in China and the influential factors. Economic Research Journal, (1): 33-44. (in Chinese)

$\mathrm{Xu}$ Yong, Fan Jie, 2014. Index system for regional development disparity measurement. Progress in Geography, 33(9): 1159-1166. (in Chinese) 